\title{
L'iconographie et le symbolisme du palmier dattier dans l'Antiquité (Proche-Orient, Égypte, Méditerranée orientale)
}

Iconography and symbolism of the date palm in Antiquity (Near East, Egypt, Eastern Mediterranean)

\section{Fanny Michel-Dansac et Annie Caubet}

\section{OpenEdition}

Journals

Édition électronique

URL : http://journals.openedition.org/ethnoecologie/1275

DOI : 10.4000/ethnoecologie. 1275

ISSN : 2267-2419

Éditeur

Laboratoire Eco-anthropologie et Ethnobiologie

Référence électronique

Fanny Michel-Dansac et Annie Caubet, «L'iconographie et le symbolisme du palmier dattier dans

I'Antiquité (Proche-Orient, Égypte, Méditerranée orientale) », Revue d'ethnoécologie [En ligne], 4 | 2013,

mis en ligne le 07 janvier 2014, consulté le 30 avril 2019. URL : http://journals.openedition.org/ ethnoecologie/1275; DOI : 10.4000/ethnoecologie.1275

Ce document a été généré automatiquement le 30 avril 2019

\section{cc)}

Revue d'ethnoécologie est mis à disposition selon les termes de la licence Creative Commons Attribution - Pas d'Utilisation Commerciale - Pas de Modification 4.0 International. 


\title{
L'iconographie et le symbolisme du palmier dattier dans l'Antiquité (Proche-Orient, Égypte, Méditerranée orientale)
}

\author{
Iconography and symbolism of the date palm in Antiquity (Near East, Egypt, \\ Eastern Mediterranean)
}

Fanny Michel-Dansac et Annie Caubet

\section{Introduction}

1 De la Mésopotamie à l'Égypte, du Levant à la Grèce, le palmier constitue un des motifs végétaux les plus souvent représentés dans l'Antiquité, le seul dont l'iconographie peutêtre identifiée grâce à sa morphologie et son allure bien spécifiques ${ }^{1}$.

2 Parmi les espèces de palmiers présentes dans ces différentes aires géographiques Phoenix dactylifera ou palmier dattier, Phoenix theophrastii ou palmier de Crète, Hyphaene thebaica ou palmier-doum, Chamaerops humilis ou palmier nain, Medemia Argun ou palmier argoun -, le palmier dattier a donné lieu à l'iconographie la plus abondante, iconographie à laquelle nous consacrons cet article.

3 Il n'est pas de notre compétence de traiter de l'origine et de la date de domestication du Phoenix dactylifera. La zone de culture est assez bien délimitée car la plante a des besoins en eau et en ensoleillement très élevés - ce que traduit l'adage populaire arabe : «le dattier vit les pieds dans l'eau et la tête au feu du ciel ». Par conséquent, seules certaines zones de l'aire géographique qui nous occupe - principalement les oasis de Mésopotamie et d'Égypte - ont pu développer sa culture sur une grande échelle. Ces civilisations cultivèrent très tôt l'arbre, élaborèrent un vocabulaire très large pour désigner la plante et son usage et furent à l'origine d'une imagerie dont nous allons tenter d'évoquer la richesse. 
4 En dehors de ces zones de culture, notamment à Chypre et en Grèce, l'imagerie du palmier a été également très variée. Chaque civilisation a développé autour du palmier dattier un ensemble de significations qui témoignent des convergences et des contacts entre les peuples autant que des spécificités propres à chacune.

\section{Le motif dans les différentes aires géographiques}

\section{Mésopotamie et Iran du sud-ouest}

5 La culture du dattier couvre principalement un territoire allant de la partie marécageuse du sud de la Basse Mésopotamie à la Babylonie et une partie de la " petite Mésopotamie ", comme on désigne souvent le Khuzistan ou Iran du sud-ouest. De nombreux textes suméro-akkadiens, à caractère économique et littéraire, rendent compte de la position éminente du dattier dans cette région (Landsberger 1967; Joannès 2001, 625). En témoigne un classique dialogue littéraire opposant le palmier au tamaris, dans lequel le premier affirme sa prééminence sur les autres végétaux (André-Salvini 2008, 327). Le motif du dattier occupe une place importante dans l'iconographie mésopotamienne (Danthine 1939) où il apparaît dès la seconde moitié du $\mathrm{III}^{\mathrm{e}}$ millénaire en pays de Sumer. Il est représenté de façon très reconnaissable, avec des régimes de dattes, et semble très tôt lié à des notions de fertilité, d'agriculture prospère et de renouvellement de la végétation. Un bon exemple est le sceau-cylindre akkadien du scribe de Zaganita (Benoit 2003, 266-267), daté vers 2250 av. J.-C., mettant en scène une assemblée de dieux œuvrant au renouveau de la nature, à l'équinoxe du printemps. Ce renouveau semble symbolisé par le grand palmier chargé de dattes, image d'une prospère et féconde végétation qui se renouvelle chaque année grâce à l'action des dieux (Figure 1). Un vase en stéatite d'Iran du musée du Louvre (Gros de Beler \& Marmiroli 2008, 46-47), daté du III millénaire, présente une frise de palmiers chargés de lourds régimes de dattes: on le comparera, pour sa signification, aux frises d'épis de blé évoquant la fertilité des terres.

Figure 1. Sceau-cylindre du scribe Zaganita. Vers 2250 av. J.-C. Paris. Musée du Louvre

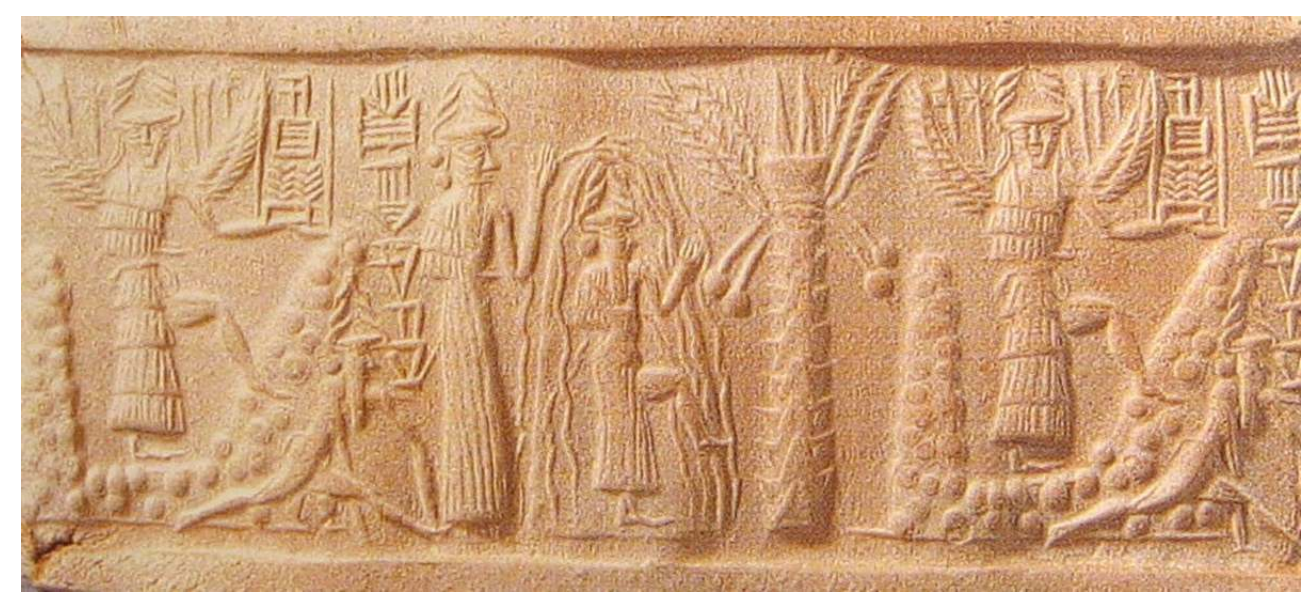

(c) Benoit 2003, fig. 117

Source de richesse pour le royaume, le palmier dattier est lié à la figure royale qui apparaît comme la représentante du pouvoir divin sur terre et la garante de la prospérité du pays, donc de l'abondance des récoltes. Une stèle néo-sumérienne trouvée à Suse (André-Salvini 2008, fig. 5), datée de la fin du $\mathrm{III}^{\mathrm{e}}$ millénaire, montre le personnage royal 
effectuant une libation au dessus d'un vase ou autel d'où jaillissent une grande palme et deux régimes de dattes, face au dieu Sin représenté assis sur un trône. Le palais royal de Mari, au XVIII ${ }^{\mathrm{e}}$ siècle av. J.-C., accorde au palmier dattier un rôle symbolique important. $\mathrm{Au}$ centre de la cour dite du Palmier, ouvrant sur la salle du trône, se dressait un grand palmier de métal. Le mur méridional de cette cour était orné d'une peinture, dite de l'Investiture, figurant la remise des insignes du pouvoir au roi par la déesse Ishtar, dans une scène encadrée par deux dattiers (Margueron 2000).

\section{L'Égypte}

7 Très répandu en Égypte pendant l'Antiquité, Phoenix dactylifera (bnr) prête son nom à divers toponymes (Baum 1988, 103-104). Selon l'inventaire des arbres cultivés dans le jardin d'Ineni (Baum 1988, 1-17), notable thébain qui vécut au début de la XVIII ${ }^{\mathrm{e}}$ dynastie, les palmiers (au dattier s'ajoutent ici deux espèces, le palmier doum et le palmier argoun) représentaient à eux seuls quasiment les deux tiers des arbres plantés dans son domaine. Le dattier acquit une importance culturelle et symbolique très forte dans la religion égyptienne.

8 Son image apparaît dès la fin du $\mathrm{IV}^{\mathrm{e}}$ millénaire (Wallert 1962). Une tablette en ivoire du roi Djer, datant de la première dynastie, représente le sanctuaire de Bouto, ville située dans le delta du Nil, figuré par des palmiers simplifiés alternant avec des chapelles, de part et d'autre d'un cours d'eau (Hugonot 1989, fig. 183 ; Servajean 1999, 228). La palmeraie de Bouto met en œuvre toute une symbolique attachée au palmier où la régénération végétale correspond à la renaissance du défunt (Servajean 1999). Cette symbolique est vraisemblablement à rechercher dans les caractéristiques botaniques du palmier dattier, à savoir sa capacité à se régénérer et à indiquer l'eau, à faire de l'ombre et à fournir de la nourriture. Cette fonction funéraire du bosquet de palmiers procède vraisemblablement d'une conception analogue à celle que l'on retrouvera dans les très nombreuses représentations de jardins funéraires du Nouvel Empire. Citons comme exemple les jardins des tombes thébaines de Sennedjem et de Rekhmirê (Gros de Beler \& Marmiroli 2008, 129-134) dans lesquelles plusieurs personnages - propriétaires de la tombe, serviteurs, prêtres - travaillent au jardin, au halage des barques et aux travaux des champs (Figure 2). 
Figure 2. Détail d'une peinture murale thébaine. Tombe de Sennedjem $\left(n^{\circ} 1\right) . x I X^{e}$ dynastie $\left(X I I I^{e}-x I^{e}\right.$ siècle av. J.-C.)

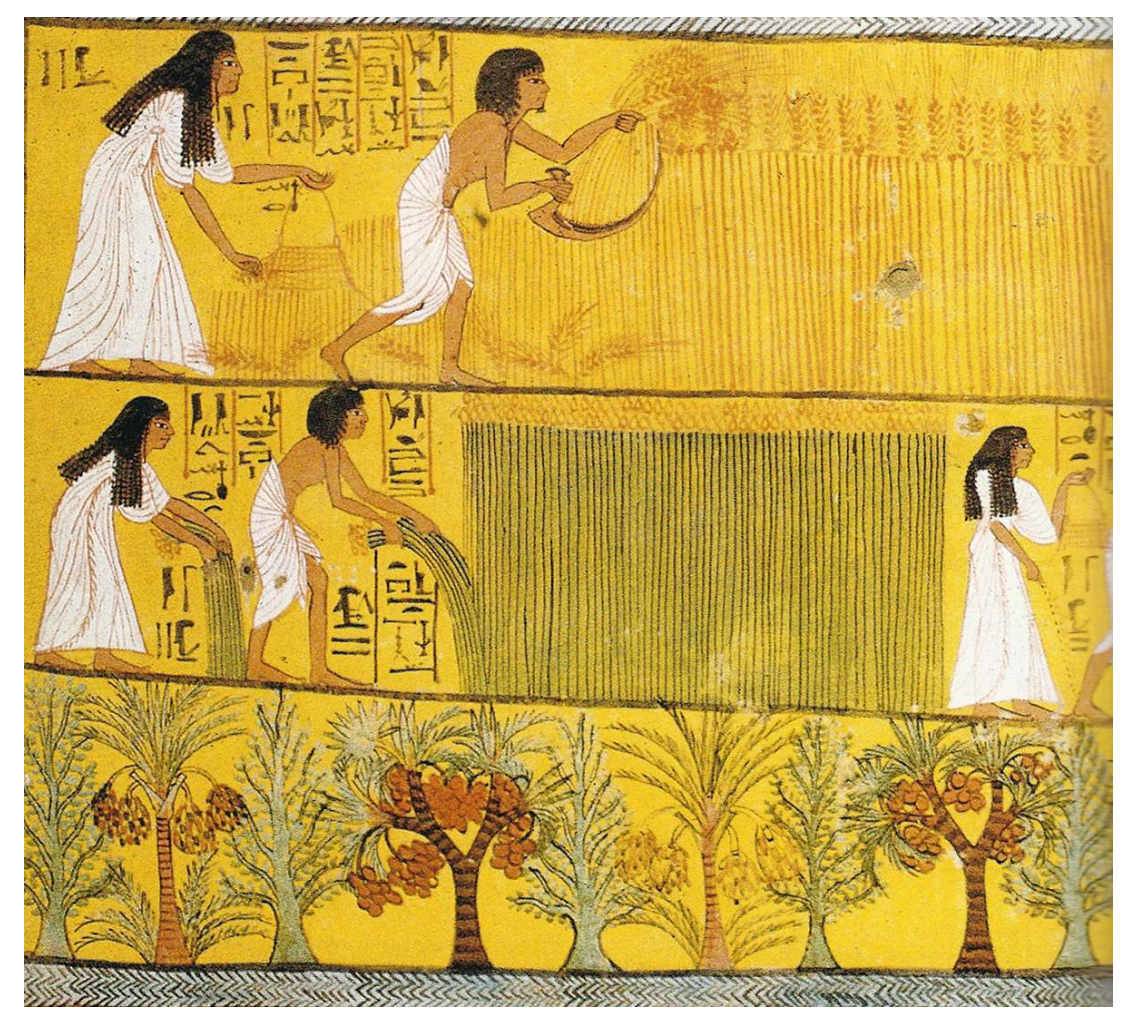

(C) Gros de Beler \& Marmiroli 2008, fig. p. 130

\section{Le Levant}

Le palmier dattier n'est productif que dans les oasis, principalement de Palmyre, de Jéricho et Ein Gedi sur la mer Morte. Tamar en hébreu, il apparaît à plusieurs reprises dans les textes de l'Ancien Testament, rédigés au $\mathrm{I}^{\mathrm{er}}$ millénaire, dans des noms de localités géographiques (Livre des Rois, 1, 9, 18 ; Genèse, 14, 7 ; Chroniques 2, 20, 2 ; Deutéronome, 34, 3 ; Chroniques 2, 28, 15). Pour les Grecs et les Romains, la Judée était célèbre pour ses palmeraies (Strabon, XIV, 2, 41 ; Théophraste, II, 6, 8 ; Pline, XIII, 44).

Le motif iconographique est développé dès le Bronze Moyen et Bronze Récent, un peu à l'écart des zones de culture, sur les grands sites proches du littoral méditerranéen. Il est représenté sans fruits - jusqu'à une époque tardive, vers le viI ${ }^{\mathrm{e}}$ siècle av. J.-C. - et de façon assez schématisée. Il est le plus souvent flanqué d'animaux, oiseaux ou quadrupèdes. C'est, transposé à l'image du palmier, le motif de l'arbre stylisé encadré d'animaux antithétiques, de tradition ancienne au Proche-Orient pour symboliser l'équilibre de la nature. Sur une cruche de Megiddo, du Xve siècle av. J.-C., sont représentés deux oiseaux au sol de part et d'autre d'un palmier (Kepinski 1982, $n^{\circ}$ 775). Une jarre de Megiddo, du XIV siècle av. J.-C., est ornée de deux chèvres cabrées et affrontées de part et d'autre du motif (Kepinski 1982, $\mathrm{n}^{\circ}$ 780). Oiseaux ou chèvres composent avec le palmier un tout indissociable, relevant du domaine du sacré. Le palmier peut, plus spécifiquement, être lié à la sphère d'une divinité féminine (Keel \& Uehlinger 2001, pp. 38, 59, 63). 


\section{Hors des zones de production}

11 Dans des zones où la plante ne fait pas l'objet d'une véritable culture, où les dattes atteignent mal ou pas du tout la maturité, on observe que le motif du palmier occupe cependant une place importante. Outre la Mésopotamie septentrionale et le littoral levantin, l'île de Chypre et la Grèce donnent naissance à une très riche iconographie du palmier, dès le $\mathrm{II}^{\mathrm{e}}$ millénaire. Parmi les exemples les plus remarquables, une coupe de Lapithos, datée de 1050-950 av. J.-C., est décorée d'un palmier sur lequel perchent deux oiseaux (Karageorghis, des Gagniers 1974, fig. p. 356) ; un sceau de Mycènes, daté de la seconde moitié $\mathrm{du} \mathrm{II}$ e millénaire, représente le sacrifice d'un quadrupède sous un palmier (Marinatos 1984, fig. 7) ; sur une amphore attique du peintre Exékias, datée des environs de 540 av. J.-C., figure le suicide d'Ajax sous un palmier (LIMC I « Aias I », n 104).

Une telle diffusion, touchant des régions où le dattier n'a pas de fonction économique, résulte peut-être de contacts entre peuples voisins - conquêtes, contrôle politique, voyages, mais aussi d'échanges commerciaux. De nombreux produits utiles dérivés du dattier (fruits, régimes frais ou séchées, « miel » de dattes) ont circulé, établissant très tôt un réseau de relations avec les régions hors des centres de production. Notons que Strabon (XIV, 1, 14) puis Plutarque (VIII, 4, 5) citent un chant perse qui énumère jusqu'à trois cent soixante manières d'utiliser le palmier.

Un grand nombre de civilisations antiques adoptent ainsi le palmier et se l'approprient en l'intégrant dans un vocabulaire iconographique et symbolique qui lui est propre. Certains grands thèmes symboliques apparaissent récurrents et permettent d'observer la façon dont la vision de l'arbre se manifeste dans chacune des cultures et religions.

\section{Le développement symbolique du motif}

\section{Le palmier et l'eau}

L'association de l'eau et du palmier est particulièrement développée dans le monde proche-oriental et égyptien. Cette complémentarité, fondamentale sur le plan physiologique, est évidente dans l'iconographie symbolique mésopotamienne. Sur le sceau du scribe Zaganita, déjà cité, le dattier est directement associé au dieu des eaux douces Ea, reconnaissable aux flots qui l'entourent et jaillissent d'un vase qu'il tient dans la main. Cette complémentarité de l'eau et du palmier acquiert une dimension particulièrement importante en Égypte, notamment au Nouvel Empire: des palmiers ${ }^{2}$ sont systématiquement figurés au bord des bassins et des canaux ; ils définissent, avec les sycomores, les jardins funéraires indispensables où le mort doit pouvoir se rendre pour se désaltérer. Les tombeaux étant aménagés dans le désert, hors des précieuses zones cultivables, l'image du palmier vient rappeler au mort la fraîcheur vitale de l'eau : dans la tombe de Neferhotep (Hugonot 1989 : fig. 172) le défunt s'agenouille pour se désaltérer dans un bassin encadré de dattiers.

Au Levant, politiquement et culturellement en liaison avec l'Égypte, la complémentarité de l'eau et du palmier se manifeste dans de nombreuses représentations de poissons et de flots, en forme de lignes ondulées. Un sceau syrien du Bronze Moyen (Keel 2007, fig. 94) représente deux poissons longeant le stipe du palmier; sur un bol de Gezer (Kepinski 1982, $\mathrm{n}^{\circ}$ 754) de la fin du deuxième millénaire, est visible un palmier se reflétant en miroir dans un cours d'eau (Figure 3). 
Figure 3. Bol en céramique de Gezer. Xle siècle av. J.-C.

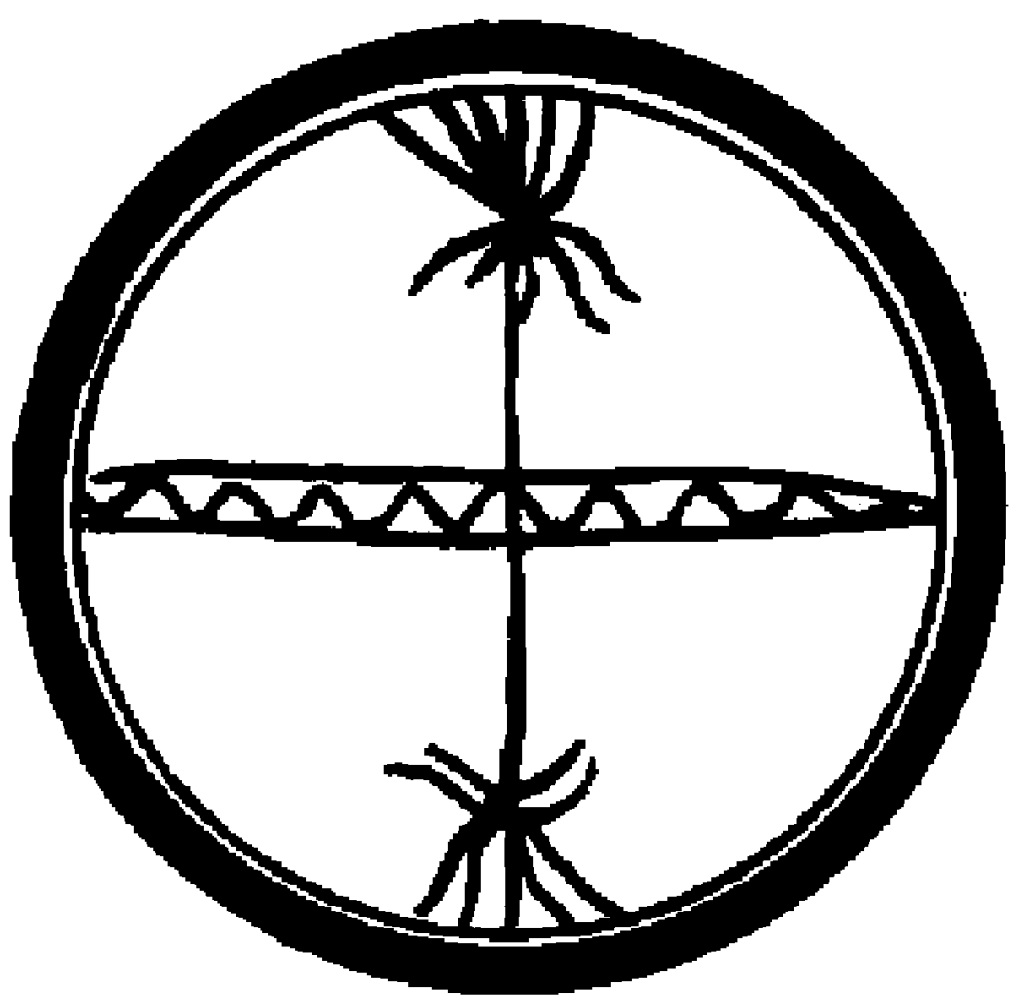

(c) Kepinski 1982, fig. $n^{\circ}$ 754. Le palmier et la datte systématique. C'est le cas en Grèce où le fruit est absent, ce qui correspond ici à une réalité botanique, ainsi qu'en témoignent les textes qui décrivent le palmier comme une plante mal acclimatée dont les fruits ne mûrissent pas ou qui n'en produit pas du tout (Théophraste II, 2, 8 ; Plutarque Propos de Table, VIII, 4,1 ; Pausanias, IX, 19, 8). palmier. En Mésopotamie, les régimes de dattes apparaissent même parfois sans le palmier. La déesse Ninhursag est souvent représentée tenant dans ses mains des régimes de dattes (Parrot 1960, fig.167 B), ces attributs signifiant ses pouvoirs sur la fécondité. Sur une stèle de Tello, datée du milieu du III ${ }^{\mathrm{e}}$ millénaire, une libation est effectuée sur un vase d'où pendent deux régimes symétriques, en l'honneur d'une divinité féminine, probablement Ninhursag (Parrot 1960, fig. 161 B).

18 En Égypte, les Textes des Pyramides (chap. 403) citent les dattes et l'eau comme élément essentiel de la nourriture du défunt. Elles figurent dans les listes d'offrandes dès la deuxième dynastie (Baum 1988, p. 95). Plus tard, les dattes constituent une offrande spécifiquement osirienne (Cauville 1980, p. 47-64).

$19 \mathrm{Au} \mathrm{IV}^{\mathrm{e}}$ siècle, Théophraste (II, 6, 7-8) note qu'à Chypre les fruits des palmiers ne mûrissent pas. Pourtant la représentation figurée des régimes de dattes est attestée dans l'île à partir du $\mathrm{VII}^{\mathrm{e}}$ siècle. Le dattier y apparaît dans des rituels de cueillette, dont la composition est très proche de celles que l'on trouve en Mésopotamie et en Égypte. Il pourvoit aux offrandes en l'honneur de divinités, sur une coupe métallique chyprophénicienne de Kourion (Culican 1982, 13-32 ; Markoe 1985 : p. 181-182) ou sur un bol de 
Kandou (Hermary 2005) où est dépeinte la cueillette de dattes associée à une scène de culte (fig. 4 et fig. 5).

Figure 4. Détail d'une coupe métallique phénicienne de Kourion. Fin du VIII ${ }^{\mathrm{e}} \mathrm{VII}{ }^{\mathrm{e}}$ siècle av. J.-C. New York, Metropolitan Museum of Art

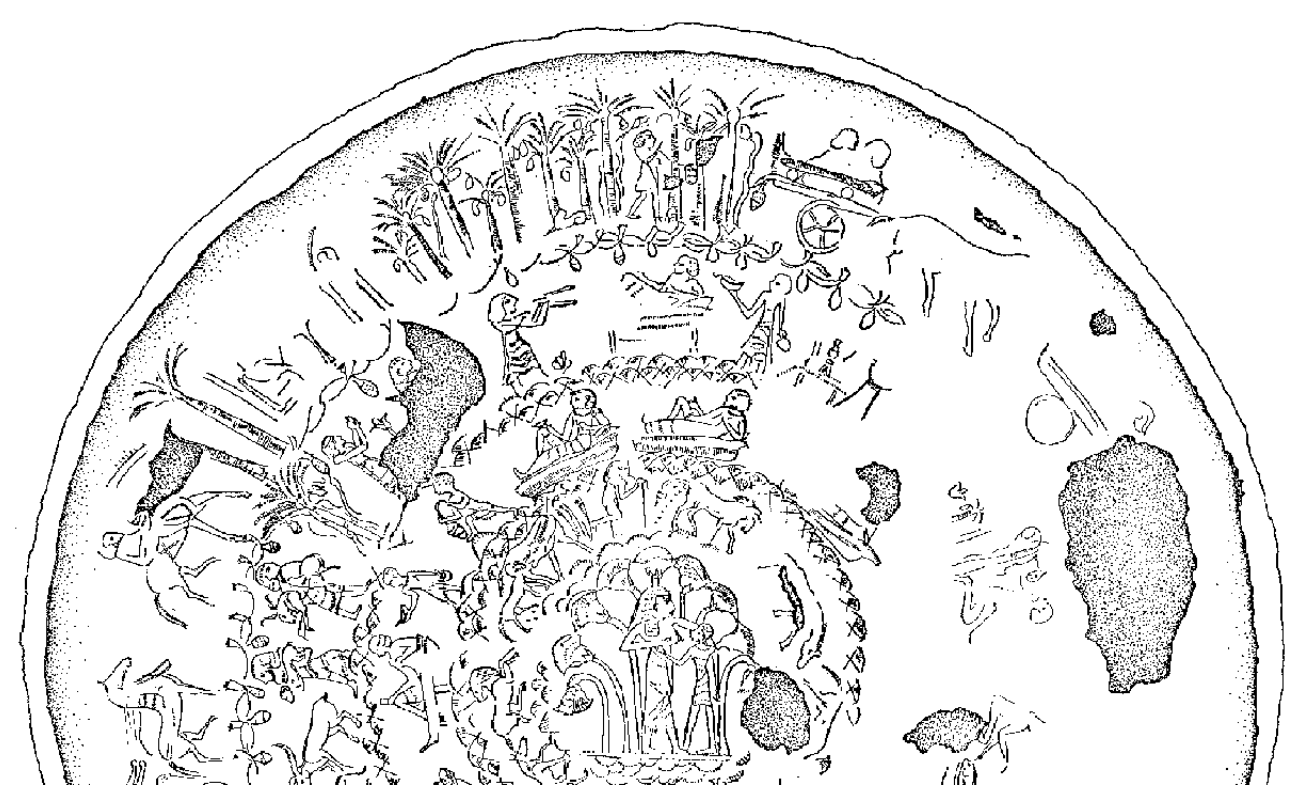

(c) Hermary 2005, fig. 5

Figure 5 : Grand bol chypriote de Kandou. Milieu du VIII ${ }^{\mathrm{e}}$-fin du VII ${ }^{\mathrm{e}}$ siècle av. J.-C. Nicosie, Cyprus Museum

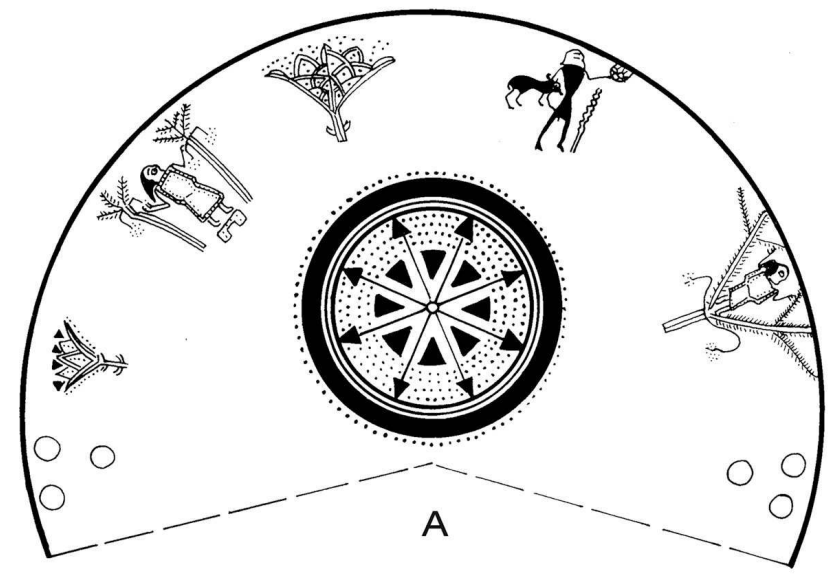

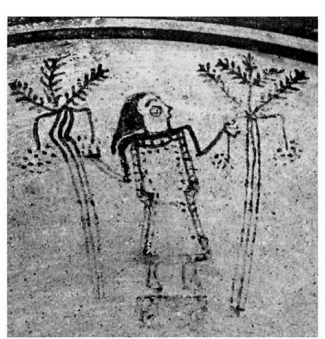

B

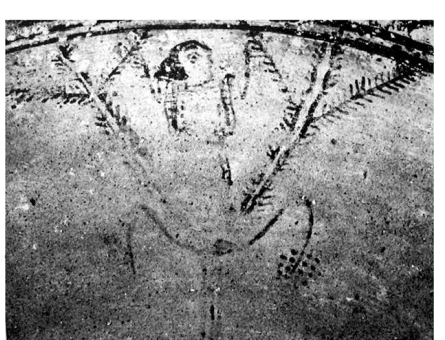

C

\section{A : DESSIN. B-C : DÉTAILS}

(C) Hermary 2005, figures 1, 2, 4 
Le palmier est par là étroitement associé à une divinité féminine nourricière, qui se manifeste parfois à l'intérieur même de l'arbre. C'est ce que l'on peut observer sur un relief égyptien de la xix dynastie (Baum 1988, 280-281), qui montre l'épiphanie de la déesse-arbre, les bras et la tête sortant d'un dattier (Figure 6) ou sur le bol chypriote de Kandou, ou la «déesse aux bras levés » apparaît dans un palmier chargé de dattes (Figure 5C).

Figure 6 : Stèle en relief d'une tombe memphite. XIX dynastie. (XIII-XII siècle av. J.-C.). Le Caire, Musée égyptien

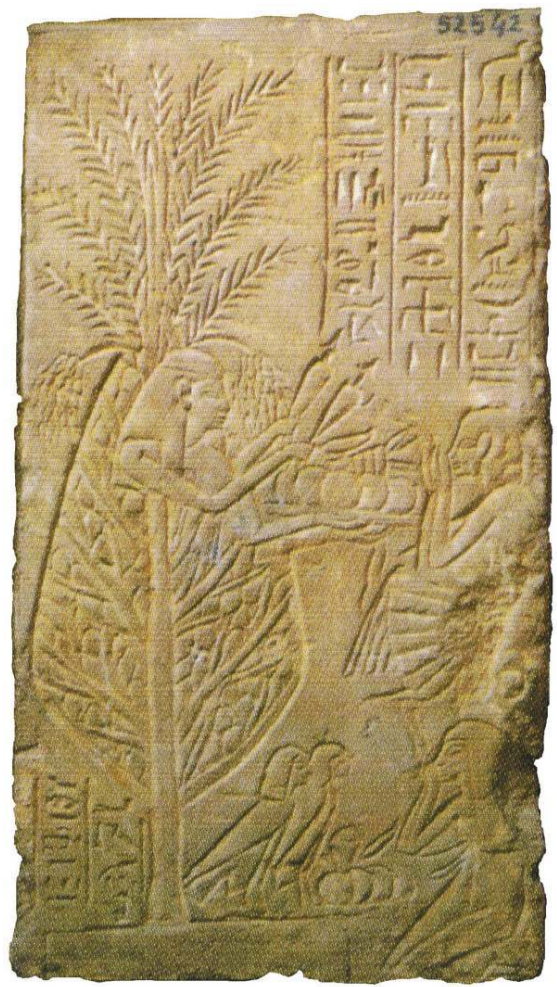

(c) Ziegler C. (Dir.), Pharaons, Institut du Monde Arabe, Paris, 2004, p. 213 n 89

\section{Le palmier et la femme}

21 Un thème notable revient dans l'ensemble des civilisations étudiées : le palmier est de façon récurrente associé à la femme (Michel-Dansac 2011, 227-252); soit dans une véritable assimilation, soit dans une démarche anthropomorphique, consistant à prêter des attributs ou des réactions humaines à l'arbre.

Les particularités organiques et morphologiques du palmier sont à l'évidence à l'origine de cette association : le caractère diö̈que du palmier (qui présente des fleurs mâles et des fleurs femelles sur des pieds séparés), les techniques de fécondation artificielle qui lui sont appliquées, ne peuvent que suggérer un fort anthropomorphisme. L'esthétique des représentations figurées du palmier a contribué à donner à cette plante une personnalité féminine. Sur ce point, la linguistique et les textes complètent très souvent la lecture des images. Le corps de la femme est, dans l'image, comparé au stipe de la plante (Figures 5C et 6) son allure et son élégance sont comparées à la verticalité et à l'élan du palmier :

Revue d'ethnoécologie, 4 | 2013 
«... tu es élancée comme le palmier...»

Cantique des cantiques (VII, 8-9)

«Ton aspect me confond! Un jour, à Délos, près de l'autel d'Apollon, j'ai aperçu

même beauté : le rejet d'un palmier qui montait vers le ciel »

L'Odyssée (VI, 162).

La pratique agricole consistant à polliniser artificiellement les palmiers femelles, connue très tôt en Basse Mésopotamie (Landsberger 1967, 18), a donné lieu à toute une symbolique associant la fertilité de l'arbre et la fécondité de la femme. Le terme akkadien désignant ce procédé de fertilisation, rakabum, désigne également (ou est emprunté à) l'acte de féconder sexuellement une femme. Un peigne en ivoire d'Assur (Caubet 2008, 115), représente les préparatifs de noces d'une jeune femme, dans laquelle l'image des dattiers fait allusion à la fécondité attendue de la principale protagoniste (Figure 7).

Figure 7 : Peigne en ivoire d'Assur. Tombe 45. XIII siècle av. J.-C. Berlin. Vorderasiatisches Museum
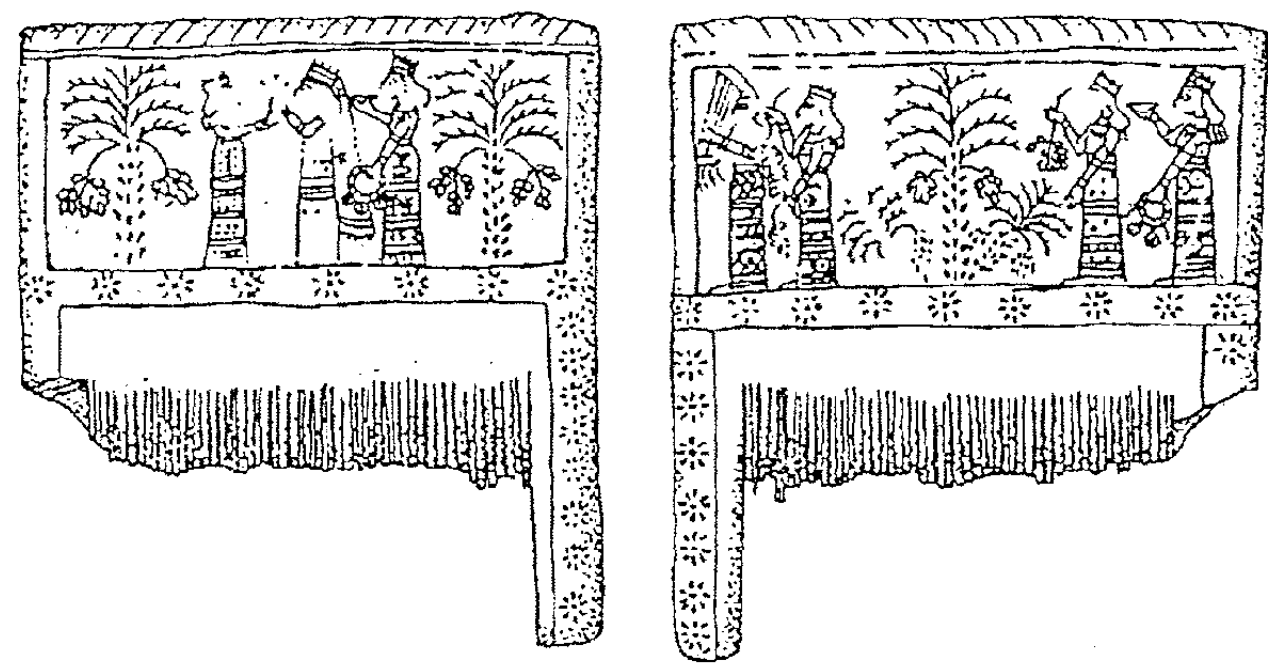

(C) CAUBET 2008, fig. 3

Le sein de la femme et à travers lui sa forme et sa fonction nourricière sont directement mis en parallèle avec la datte : les régimes de dattes sont fréquemment représentés par paire de part et d'autre du stipe. Cette analogie entre les seins et les dattes s'observe sur une scène d'allaitement d'un relief de Karatépé (Soldi \& Pedrazzi 2006, 278-279), montrant une déesse allaitant un enfant à l'ombre d'un palmier dattier (Figure 8). 


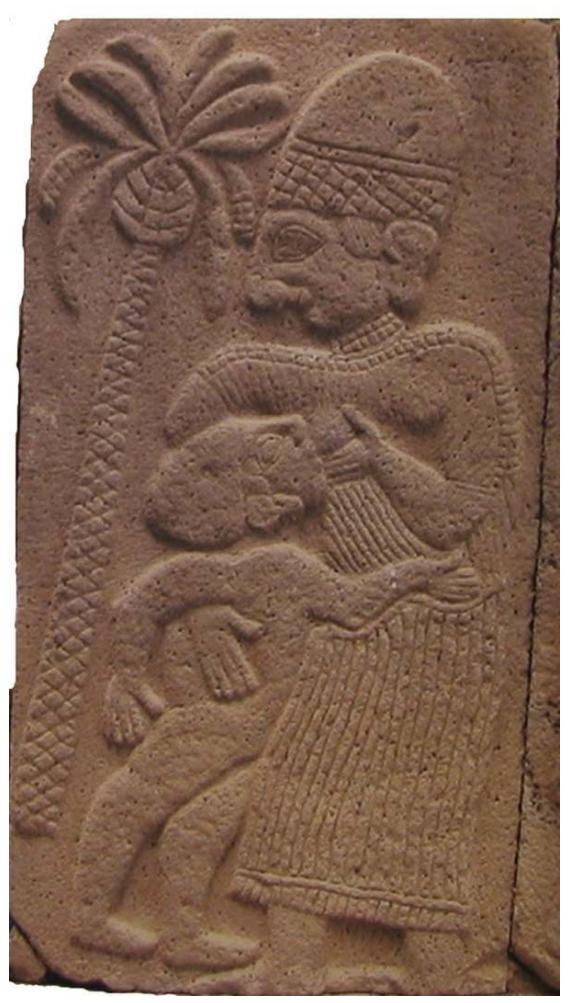

(c) Soldi \& Pedrazzi 2006, fig. 96

L'idée qu'une divinité féminine et maternelle puisse être associée au dattier ou même se manifester sous l'aspect du dattier a perduré jusqu'à une époque tardive. Dans le papyrus Jumilhac (XIV, 12), le dattier apparaît comme la manifestation d'Isis pleureuse, balançant sa ramure comme la pleureuse sa chevelure. La disposition très particulière des palmes au sommet du stipe a, en effet, suscité un rapprochement avec la chevelure féminine. Sur une hydrie attique du peintre de Kléophradès (LIMC VIII « Ilioupersis »: $\mathrm{n}^{\circ} 11$ ), le palmier se penchant précisément sur la chevelure d'une femme assise en lamentation peut évoquer une femme en pleurs secouant sa chevelure sur la ville détruite de Troie, telles les pleureuses sur le corps d'un défunt (Figure 9).

Il est remarquable qu'en Grèce, où le palmier n'a aucune valeur agricole et économique, il n'intervient, ni comme symbole nourricier, ni comme symbole de la fertilité, alors qu'un fort anthropomorphisme conduit à voir en lui l'image de la femme et de sa beauté. Dans les différentes versions du mythe de l'accouchement de Léto à Délos sous un palmier ( Hymne homérique à Apollon, 119-121; Théognis, Poèmes élégiaques I, 5-9; Callimaque, Hymne à Délos, 209-210; Le Roy 1973), le palmier joue le rôle de déclencheur dans la délivrance de la déesse, prenant le rôle que tient habituellement la sage-femme. Ce thème est parfaitement illustré sur une pyxide attique (LIMC VI « Leto », $\mathrm{n}^{\circ} 6$ ) représentant la déesse assise sur un tabouret d'accouchement et s'agrippant au stipe d'un palmier dressé devant elle (Figure 10). 
Figure 9 : Détail d'une hydrie attique à figures rouges du peintre de Kléophradès. Vers 480 av. J.-C. Naples, Museo Nazionale

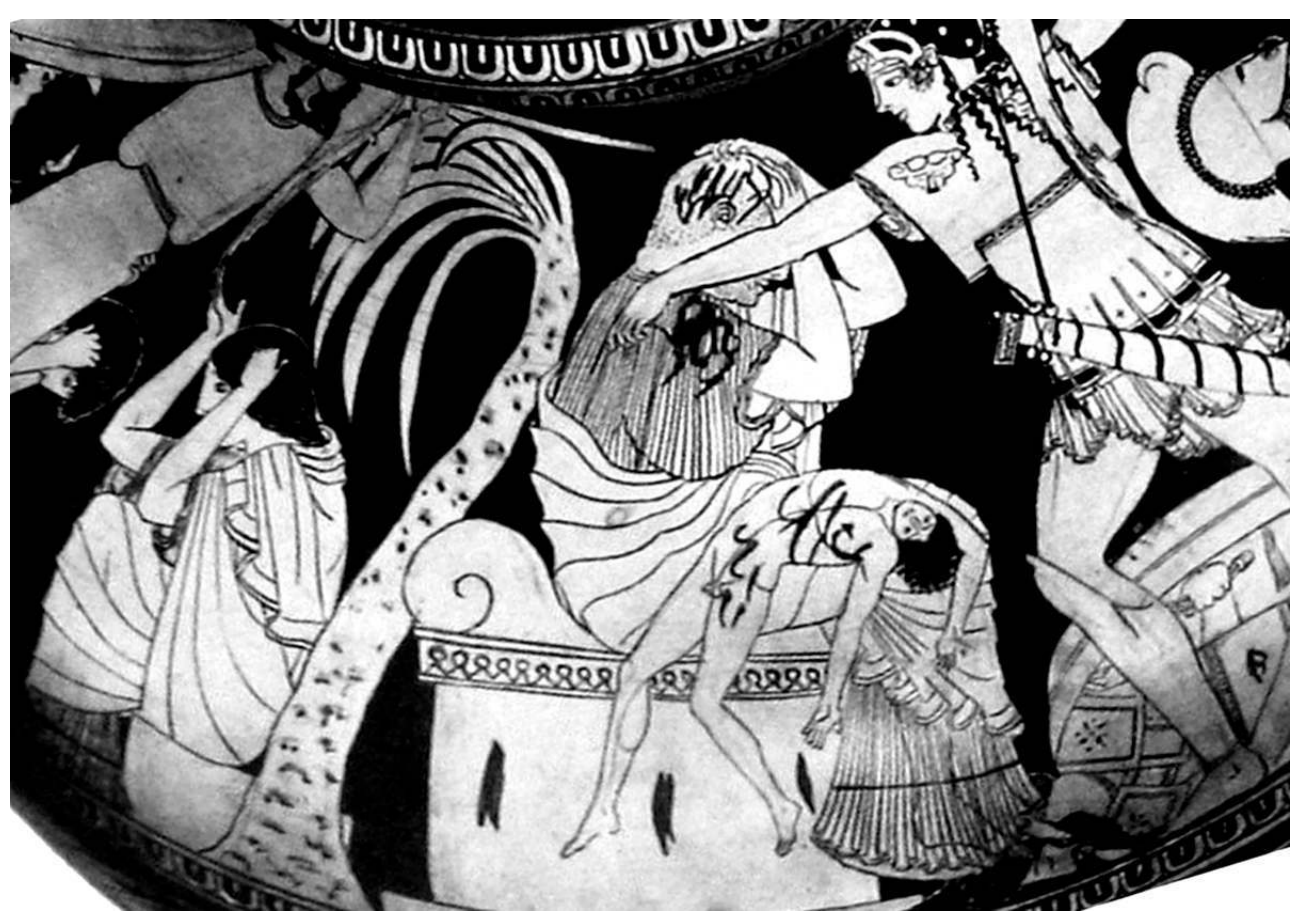

(c) LIMC VIII « llioupersis », $n^{\circ} 11$

Figure 10 : Détail d'une pyxide attique polychrome. Ive siècle av. J.-C. Athènes, Musée National

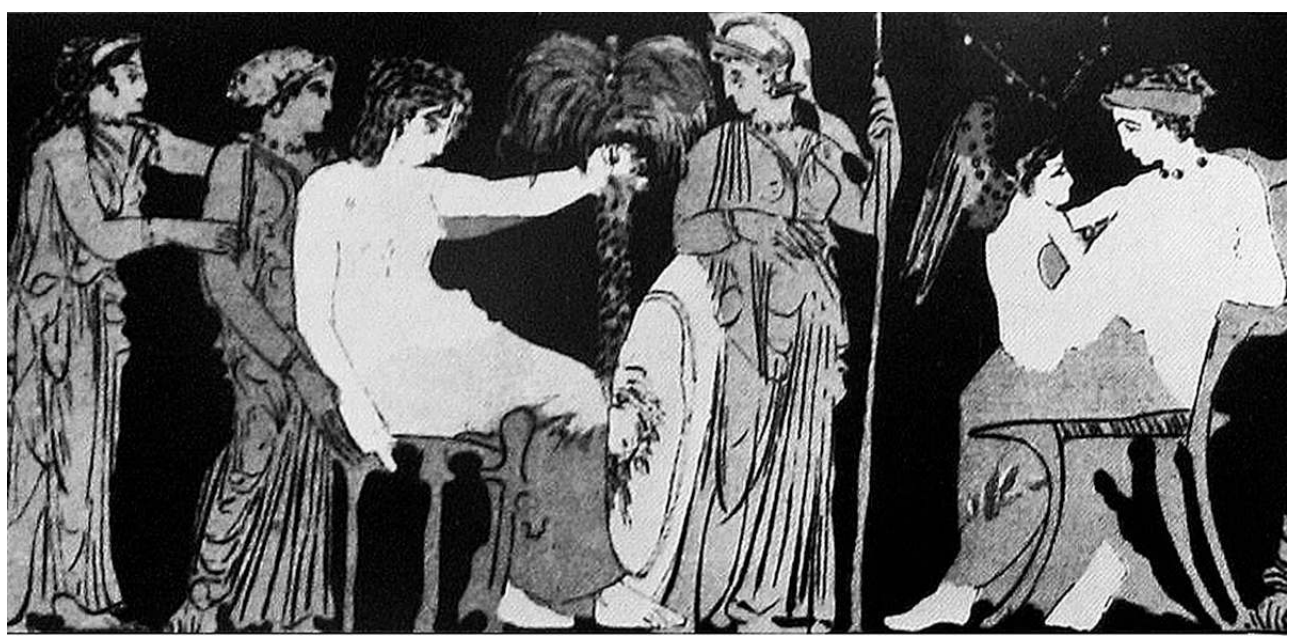

(c) LIMC VI « Leto », $n^{\circ} 6$

\section{Le palmier et l'espace de culte}

Le palmier ou la palmeraie en vient à désigner, de façon récurrente, un espace cultuel (Michel-Dansac 2011, 202-226). En Égypte, le dattier est l'arbre sacré d'une série de localités ; c'est tout particulièrement le cas de Bouto. Le bosquet constitué de dattiers associé au doum et au sycomore - caractérise les nombreux jardins funéraires et sacrés du Nouvel Empire (Figure 2). 
Plusieurs coupes métalliques chypro-phéniciennes (Markoe 1985 : fig. p. 264, p. 348) et des céramiques chypriotes archaïques de la région d'Amathonte (Hermary 2005 ; Caubet 2008) présentent une cérémonie cultuelle sous un bosquet de dattiers, servant de cadre à la cérémonie et définissant ainsi un sanctuaire en plein air (Figures 4 et 5). Dans ce cas, la présence des bosquets sacrés est probablement à rapprocher du développement de ce thème dans le monde oriental et égyptien.

En Grèce, le palmier, représenté sans fruit, - isolé, parfois associé à un autel - désigne un sanctuaire, le plus souvent consacré aux divinités Apollon et Artémis auxquelles il est étroitement lié (Miller 1979; Sourvinou-Inwood 1985; Monbrun 1989), comme le sanctuaire de Délos (Figure 11), mais également celui d'Apollon à Delphes (LIMC VII, « Python », n 3) ou celui d'Artémis à Brauron (Kahil 1977, pl. 18).

Figure 11 : Amphore attique à figures noires du peintre de Rycroft. Fin du vie siècle av. J.-C. Oxford, Ashmolean Museum

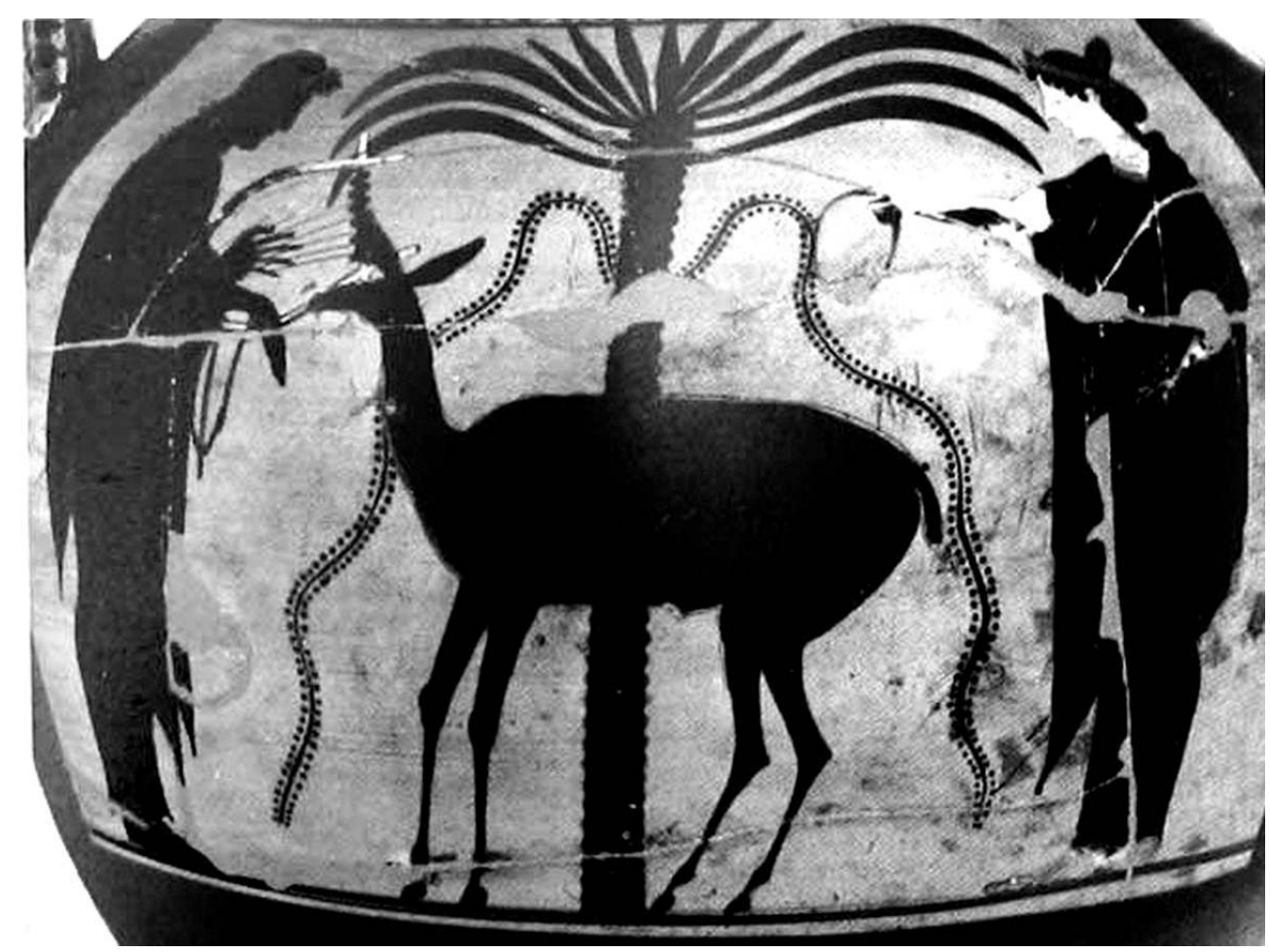

(C) LIMC II «Apollon»: n 673 b 


\section{Conclusion}

31 L'approche transversale de cette étude a tenté de mettre en avant la pérennité de thèmes véhiculés par le motif du palmier dattier, observant au passage des différences qui tiennent au tempérament artistique et idéologique des différentes cultures où s'épanouissent ces motifs symboliques.

Le palmier, loin de s'être banalisé, d'avoir été ramené à la simple image d'un exotisme oriental, à une image esthétique et décorative, est resté chargé jusqu'à nos jours d'une symbolique religieuse puissante. Ce motif s'est ainsi imposé sur une aire géographique très vaste, dépassant largement les terres d'élection de la plante, sur la longue durée; d'autres motifs végétaux, tel le sycomore en Égypte ou l'olivier en Grèce ou bien d'autres motifs végétaux « réels » et « imaginés » ont connu un succès considérable, mais aucun sans doute n'a cette puissance d'évocation exceptionnellement riche.

\section{BIBLIOGRAPHIE}

Textes antiques et médiévaux

Bible (La), L'Ancien Testament. Paris, les éditions du Cerf, 1975.

Callimaque, Épigrammes et Hymnes, texte établi et traduit par E. Cahen 1948. Paris, Les Belles Lettres.

Homère, Odyssée, texte établi et traduit par V. Bérard. Paris, Les Belles Lettres : tomes I, chants IVII, 1947 ; tome II, chants VIII-XV, 1953.

Hymnes homériques, texte établi et traduit par J. Humbert 1951. Paris, Les Belles Lettres.

Pausanias, Description de la Grèce, livres IX-X, texte traduit par W. H. S. Jones 1955, Cambridge Ma, Londres, Heinemann LTD et Harvard University Press.

Pline l'Ancien, Histoire Naturelle, livre XIII, texte établi et traduit par A. Ernout 1956. Paris Les Belles Lettres.

Plutarque, Propos de Table (livres VII-IX), texte établi et traduit par F. Frazier \& J. Sirinelli 1996. Paris, Les Belles Lettres.

Strabon, Géographie, livre XIV, texte établi et traduit par H. L. Jones 1969, Cambridge Ma., Londres, W. Heinemann LTD et Harvard University Press.

Théognis, Poèmes élégiaques, texte établi et traduit par J. Carrière 1948, Paris, Les Belles Lettres. Théophraste, Recherches sur les plantes, texte établi et traduit par S. Amigues 1989. Paris, Les Belles Lettres : tome I, livres I-II, 1988 ; tome II, livres III-IV.

Ouvrages modernes

André-Salvini B. (Dir.) 2008 - Babylone. Paris, Hazan/Musée du Louvre. 
Baum N. 1988 - Arbres et arbustes de l'égypte ancienne. La liste de la tombe thébaine d'Ineni ( $\left.n^{\circ} 81\right)$. Leuven, éditions Peeters. (Orientalia Lovaniensia Analecta ; 31)

Benoit A. 2003 - Art et archéologie : Les civilisations du Proche-Orient ancien. Paris, école du Louvre / Réunion des Musées nationaux. (Manuels de l'école du Louvre)

Caubet A. 2008 - Un banquet champêtre à Amathonte. In Bouet A. (Ed.), D'Orient et d'Occident, mélanges offerts à Pierre Aupert. Mémoires 19, Bordeaux : 113-117.

Cauville S. 1980 - Une offrande spécifique d'Osiris : le récipient de dattes. Revue d'égyptologie 32 : 47-64.

Culican W. 1982 - Cesnola bowl 4555 and other Phoenician bowls. Rivista di Fenici : 13-32.

Danthine H. 1939 - Le palmier-dattier et les arbres sacrés dans l'iconographie de l'Asie occidentale ancienne. Journal des savants 3 (1) : 133-134.

Gros de Beler A. \& Marmiroli B. 2008 - Jardins et paysages de l'Antiquité. Mésopotamie et égypte. Actes Sud, Arles.

Hermary A. 2005 - Scènes de culte originales sur un vase chypriote archaïque. In Spano Giammellaro A. (Ed.), Atti del V Congresso Internazionale di Studi Fenici e Punici, Marsala-Palermo, 2-8 ottobre 2000, Palerme : 171-179.

Hugonot J.C. 1989 - Le jardin dans l'égypte ancienne. Publications universitaires européennes, série XXXVIII, vol. 27. Frankfurt-am-Main.

Joannès F. 2001 - Palmier dattier. In Joannès F. (Dir.), Dictionnaire de la civilisation mésopotamienne. Paris, Robert Laffont : 624-626.

Kahil L.G. 1977 - L’Artémis de Brauron. Antike Kunst 20 : 86-98.

Karageorghis V. \& Gagniers J. des 1974 - La céramique chypriote de style figuré, âge du fer, 1050-500 av. J.-C. Consiglio nazionale delle richerche, Instituto per gli studi Micenei ed Egeo-Anatolici. Rome, Ed. dell'Ateneo e Bizzarri. (Biblioteca di antichità cipriote).

Karageorghis V. \& Gagniers J. des 1979 - La céramique chypriote de style figuré, âge du fer, 1050-500 av. J.-C. Supplément. Consiglio nazionale delle richerche, Instituto per gli studi Micenei ed EgeoAnatolici. Rome, Ed. dell'Ateneo e Bizzarri. (Biblioteca di antichità cipriote).

Keel O. \& Uehlinger C. 2001 - Dieux, déesses et figures divines. Les sources iconographiques de l'histoire de la religion d'Israël. Paris, Les Éditions du Cerf.

Keel 0. 2007 - L'éternel féminin : une face cachée du Dieu biblique. Genève, Labor et Fides.

Kepinski C. 1982 - L'arbre stylisé en Asie Occidentale. Paris, Éditions Recherche sur les civilisations. Landsberger B. 1967 - The Date Palm and its by-products according to the cuneiform sources. Weidner, Graz, Archiv für Orientforschung.

Le Roy C. 1973 - La naissance d'Apollon et les palmiers déliens. études déliennes, Bulletin de Correspondance Hellénique, supplément I : 263-286.

LIMC : Lexicum Iconographicum Mythologicae Classicae. Zürich et Munich, Artémis Verlag.

Luckenbill D.D. 1989 - Ancient records of Assyria and Babylonia vol. II, $2^{\text {nd }}$ ed., Londres.

Margueron J.C. 2000 - De Mari à Délos, un lien: le palmier. Ktema 25 : 55-63.

Marinatos N. 1984 - The Date Palm in Minoan Iconography and Religion. Opuscula Atheniensia 15 : 115-122. 
Markoe G. 1985 - Phoenician Bronze and Silver Bowls from Cyprus and the Mediterranean. Berkeley, Los Angeles, University of California Press.

Michel-Dansac F. 2011 - L'iconographie du palmier dans la Méditerranée antique : diffusion et sens du motif. Thèse de doctorat de l'Université d'Aix-Marseille.

Miller H.F. 1979 - The Iconography of the palm in Greek art : significance and symbolism. Thèse de doctorat, University of California, Berkeley.

Monbrun P. 1989 - Artémis et le palmier dattier. Pallas 35 : 69-93.

Parrot A. 1960 - Sumer. L'univers des formes. Paris, Gallimard.

Parrot A. 1961 - Assur. L'univers des formes. Paris, Gallimard.

Servajean F. 1999 - Enquête sur la palmeraie de Bouto (I). Les lymphes d'Osiris et la résurrection végétale. In Aufrère S. (Dir.), Encyclopédie religieuse de l'univers végétal. Croyances phytoreligieuses de l'Égypte ancienne. Montpellier, Publications de l'Université Paul Valéry, Montpellier 3 : 227-247. (Orientalia Monspeliensia ; 15).

Soldi S. \& Pedrazzi T. 2006 - L'art phénicien et du Moyen-Orient. La grande Histoire de l'Art. Paris, Mediasat.

Sourvinou-Inwood C. 1985 - Altars with palm trees, palm trees and parthenoi. Bulletin of the Institute of Classical Studies $32: 125-146$.

Wallert I. 1962 - Die Palmen im Alten Ägypten. Eine Untersuchung ihrer praktischen, symbolischen und religiösen Bedeutung. Berlin, Verlag Bruno Hessling. (Münchner Ägyptologische Studien ; 1)

Ziegler C. (Dir.) 2004 - Pharaons, Paris, Institut du Monde Arabe.

\section{NOTES}

1. Pour les botanistes modernes les palmiers ne sont pas des arbres. Cependant, les civilisations antiques les considéraient tels, aussi nous avons conservé ce terme, dans la mesure où nous esquissons ici son histoire culturelle.

Étant donné que notre étude comprend les représentations de palmiers (Phoenix) sans fruit du monde grec, nous préférons employer, dans certains cas, le terme de palmier à celui de dattier.

2. Ces images comprennent également des palmiers doums.

\section{RÉSUMÉS}

Le palmier dattier (Phoenix dactylifera) occupe une place importante dans l'idéologie des civilisations anciennes. Cet article s'intéresse à la naissance et au développement du motif, dès la fin du IV millénaire av.J.-C., dans les grandes zones de production agricole (oasis de Mésopotamie et d'Égypte) et à sa diffusion vers le monde méditerranéen oriental où le dattier, sans valeur économique notable, conserve ou se voit approprier cependant des aspects symboliques multiples. Certains thèmes récurrents permettent d'observer la façon dont la vision 
de l'arbre se manifeste dans chacune des cultures et religions : associé à l'eau, à la femme, le palmier en vient à désigner l'espace de culte.

The date palm (Phoenix dactylifera) plays an important role in the ideologies of past civilisations. This paper addresses the origin and development of the motif in the vast zones of agricultural production (oases of Egypt and Mesopotamia) from the late 4th millennium BC, as well as its diffusion towards the Eastern Mediterranean world where the date palm, although deprived of real economic value, still retains or acquires new and multiple symbolic significations. The recurrence of certain symbolic themes allows us to note how the plant is perceived in different cultural and religious contexts: associated with water and women, the palm tree becomes a symbol for the ritual space.

\section{INDEX}

Index géographique : Méditerranée, Proche-Orient, Mésopotamie, Levant, Iran, Égypte, Grèce Keywords : iconography, date palm, date, fertility, sacred grove, Near East, Egypt

Mots-clés : iconographie, palmier dattier, datte, fécondité, bosquet sacré, Proche-Orient, Égypte

\section{AUTEURS}

\section{FANNY MICHEL-DANSAC}

Aix-Marseille Université/Centre Camille Jullian : Maison Méditerranéenne des Sciences de l'Homme

5 rue du Château de l'Horloge, BP 647

13094 Aix-en-Provence

fannymd@wanadoo.fr

ANNIE CAUBET

Conservateur honoraire du Patrimoine

annie.caubet@free.fr 